\title{
Microbiological diagnosis of suppurative keratitis in Bangladesh*
}

\author{
GERRY WILLIAMS,' FRANK BILLSON,, RABIUL HUSAIN, ${ }^{3}$ \\ SHAHE ALAM HOWLADER, ${ }^{3}$ NAZRUL ISLAM, ${ }^{3}$ AND \\ KATHLEEN MCCLELLAN ${ }^{2}$
}

From the 'Microbiology Unit, Liverpool Hospital; ${ }^{2}$ Department of Ophthalmology, University of Sydney; ${ }^{3}$ Eye Infirmary and Training Complex of Bangladesh National Society for the Blind

SUMMARY Experience in setting up an inexpensive microbiology laboratory in the Bangladesh National Society for the Blind Eye Hospital and Training Complex at Chittagong is presented, together with the results of a pilot study to identify organisms responsible in 33 consecutive cases of suppurative keratitis in the Chittagong area of Bangladesh. Of the 33 cases 21 were positively identified by means of Gram stain and/or culture. Two-thirds of the responsible organisms were bacteria, and one-third were fungi. The bacterial causes included Streptococcus pneumoniae and Pseudomonas aeruginosa. The fungi isolated were Aspergillus fumigatus, Aspergillus ochraceus, and Fusarium solani. Among the causes of failure to diagnose the organism was chronicity of infection and previous treatment. The value of the study in the planning of future treatment rcgimens, and the implications of setting up similar relatively cheap microbiology laboratory facilities in developing countries, are discussed.

Suppurative keratitis is a common problem in Bangladesh. From a survey by the International Centre for Diarrhoeal Diseases Research, Bangladesh in the rural Matlab area it has been estimated that 230000 people are blind owing to corneal opacity and that this is mostly due to infection $(48 \%)$ or injuries $(27.5 \%$ ) (Khan MU, Haque E, Khan MR, report prepared for publication).

Suppurative keratitis in Bangladesh is known to be caused by both bacteria' and fungi. ${ }^{2}$ As ophthalmic antifungal medications are not available, fungal keratitis is inevitably progressive. In order to manage these cases microbiological diagnosis is required.

Microbiological services are frequently under considerable pressure in developing countries, both because of the numbers of patients and the problems of cost and supply of equipment and trained personnel. Microbiology tests may represent an important item of expense for patients who have to pay for tests done by government hospitals, which normally restrict their services to their own patients.

"A pilot study conducted in Chittagong. Bangladesh, in July, August. Scptember, 1983.

Correspondence $10 \mathrm{Dr}$ G Williams, Microbiology Department. Liverpool Hospital, Box 103. Liverpool. New South Wales 2170 , Australia.
The Eye Infirmary and Training Complex of the Bangladesh National Society for the Blind (BNSB) is a 120-bed eye hospital at Chittagong which has a continual requirement for microbiological diagnosis, particularly of suppurative keratitis. New patients with suppurative keratitis are seen every day at the BNSB Eye Hospital in Chittagong and in the seven smaller BNSB Base Hospitals in different regions of Bangladesh. These infections are serious problems which threaten eyesight and require hospitalisation for periods averaging 10 weeks (Das $A$, personal communication). As a result half the eye hospital beds are occupied by patients with frequently intractable infections, particularly during the monsoon period. At harvest time up to 12 patients are seen on some days with corneal injuries and infections related to harvesting or husking rice, and these patients cannot all be admitted. A restricted number of beds imposes limitations on treatment, and many cases are treated as outpatients, it being accepted that the outcome is often hopeless regardless of treatment, particularly in chronic cases suspected of being due to fungi.

A pilot study was undertaken in August 1983 and 1984 to study the aetiology and management of suppurative keratitis, involving the setting up of a 


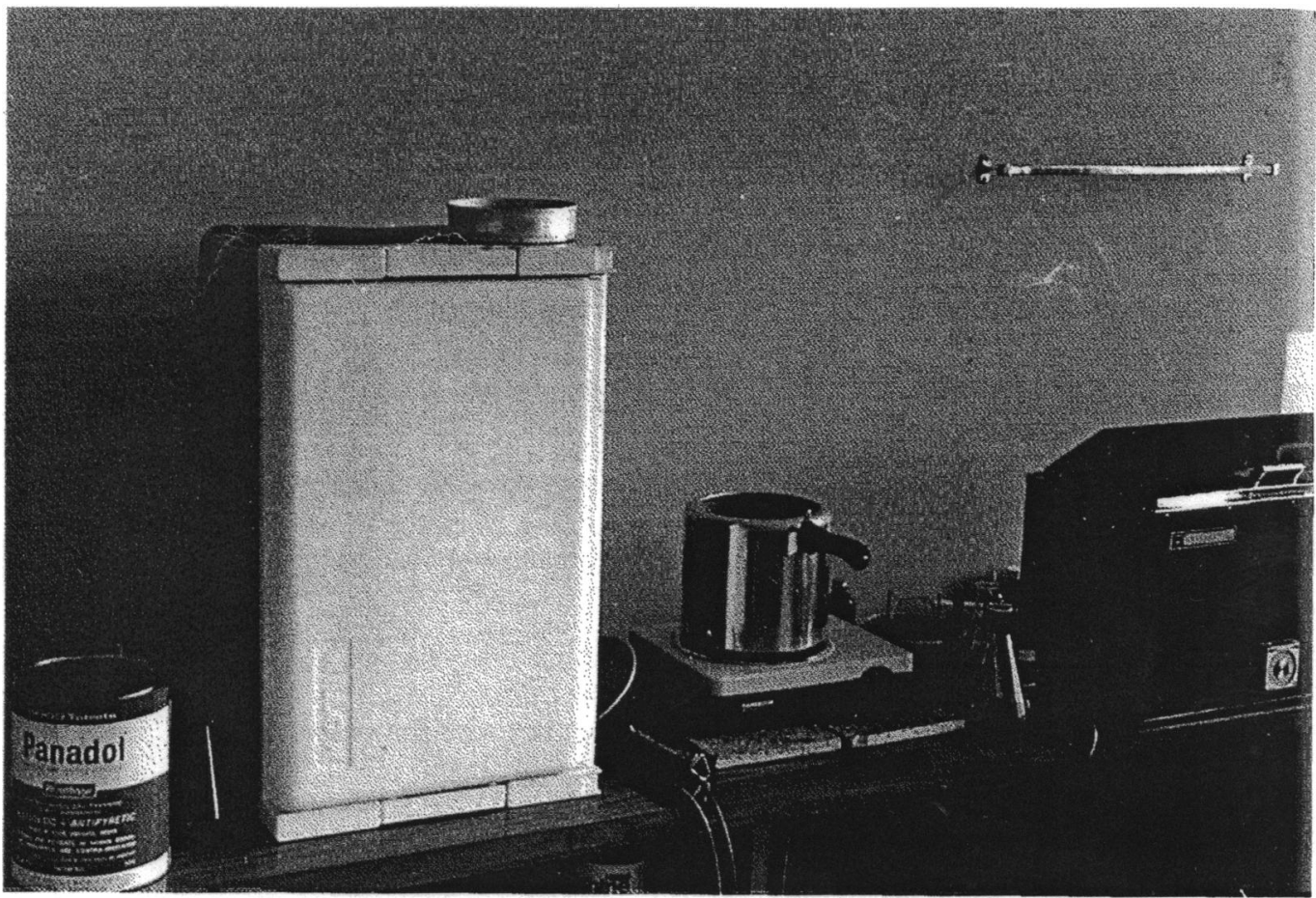

Fig.1 Microbiology Laboratory with some of the equipment. From left to right-candle tin used for $\mathrm{CO}_{2}$-dependent culture; cheap plastic ice box modified with heating element to make an incubator; pressure cooker on a portable electric hot plate; glassware; constant temperature water bath on far right.

basic microbiology laboratory using cheap readily transportable materials costing a total of 2000 Australian dollars.

\section{Material and methods}

\section{EQUIPMENT}

The following equipment was purchased in Australia and transported to Bangladesh by courtesy of Thai International Airlines, who made no charge for their services. Some of the laboratory equipment is illustrated in Fig. 1.

An incubator was constructed from a lightweight plastic cooling box with a hinged door (Vacationer made by Willow, Australia). The Anax Company assembled a 300 watt mica heating element controlled at $36^{\circ} \mathrm{C}$ by a solid state thermostat and monitor (PM4 made by RKC) with a second thermostat (Robertshaw) set to cut out at $38^{\circ} \mathrm{C}$ as a back-up. A pressure cooker was heated on a portable hot plate (Kambrook) to autoclave culture media and other materials. A binocular microscope with built-in illumination (Olympus $\mathrm{CHB}$ ) was used. Other equip- ment included the following: Bunsen burner and butane canisters (Pongrass); glass Petri dishes for agar cultures; McCartney bottles $(30 \mathrm{ml})$ for Sabouraud's agar slopes; reagents for Gram, Giemsa, Ziehl-Neelsen, and methenamine silver stains; beam balance and weights; conical flasks; bacteriological wire loops; thermometer; glass slides; Coplin jars; staining bottles; dehydrated culture media (Gibco); dehydrated rabbit plasma; antibiotic sensitivity test discs (Oxoid Multodiscs).

\section{STAFF}

A paramedic was selected (author $\mathrm{AH}$ ) from the hospital staff, and after nine months' experience in the laboratory was brought to Australia for 12 months' training. During the 12 months he attended full time the Microbiology Department at Liverpool Hospital, under the direction of author GW and spent time at Sydney Eye Hospital, and completed his training by spending a month with Professor Coster's Department in Flinders Medical Centre, South Australia.

During the evening while in Sydney he attended a 
course at the Sydney Technical College earning distinctions in microbiology and a certificate of competence.

\section{LABORATORY METHODS}

Stains were prepared by standard methods with distilled water supplied by Glaxo Company in Chittagong. Dry media bases were reconstituted with tap water and autoclaved in the pressure cooker for 15 minutes. Trypticase soy agar was used as recommended by the manufacturer (Gibco) with the addition of $2 \mathrm{~g}$ of yeast extract per litre (as suggested by Mr L Taylor, University of Sydney). A beam balance and set of weights was used for weighing media and stains. Blood was obtained from sheep purchased locally and grazed at the hospital. Wool over the neck was clipped and the area swabbed with alcoholic iodine. Blood was aspirated while the vein was dilated by pressure lower on the neck. Blood was defibrinated by mixing with sterile glass beads for five minutes. The beads could be separated from the fibrin and reused. Blood was added to sterilised media cooled to about $50^{\circ} \mathrm{C}$ to a proportion of about $10 \%$. For chocolate agar the media were reheated to $80^{\circ} \mathrm{C}$ after addition of blood for about five minutes or until chocolate brown. Vitox, a vitamin and amino acid supplement (Oxoid), was added to some batches to enhance isolation of Haemophilus influenzae ( $H$. aegyptius) from eye specimens. Poured plates were wrapped in plastic and stored in a refrigerator. The quality of media was checked by culture of Streptococcus pneumoniae on blood agar and Haemophilus influenzae on chocolate agar. These bacteria grew well on the respective media.

Corneal scrapings were plated directly on to agar plates and smeared on to glass slides for staining. Smears were stained routinely by Gram stain. Methenamine silver could be used to restain negative smears for improved detection of fungi.

Sabouraud's slopes were inoculated by several separate strokes to mark but not break the agar so that subsequent growth from the inoculum could be distinguished from contaminants not on the mark. ${ }^{3}$ Sabouraud's slopes were sealed with screw caps to prevent dehydration or contamination incubated at room temperature and were examined daily for three weeks.

Blood and chocolate agar in Petri dishes were inoculated and then streaked out with a sterile wire loop and incubated at $37^{\circ} \mathrm{C}$ in a candle tin.

Bacteria and fungi were identified by standard methods using colony morphology, Gram stain, and basic tests. Staph. aureus was identified by coagulase testing using human or rabbit plasma; Str. pyogenes by bacitracin sensitivity, morphology, Gram stain, and catalase; pneumococcus by optochin sensitivity; and Gram negative rods by decarboxylase, sugar fermentation, Voges-Proskauer, indole, urea and citrate tests as provided in the API identification kit $20 \mathrm{E}$ or the Microbact 12E test tray (Diagnostic Products, Adelaide, SA).

Haemophilus influenzae was identified by growth on chocolate agar and growth around combined $\mathrm{X}$ and $\mathrm{V}$ factor discs on nutrient agar. Pseudomonas aeruginosa was identified by characteristic colouring morphology, pigment production, and oxidase test. Fungi were identified by morphological criteria. Antibiotic disc diffusion sensitivity tests were performed by the calibrated dichotomous sensitivity method $^{4}$ on Sensitest agar. Gram stain was used alone for eight patients, of whom two had pneumococcal like bacteria and three had Gram-negative rods in scrapings from corneal ulcers.

\section{PATIENTS}

Thirty-three patients with corneal ulceration were examined over a period of three months. Histories were taken, and the eyes examined with the aid of a slit-lamp. Five of the patients had been in hospital for an average of 3.5 weeks each.

\section{CLINICAL METHODS}

Specimens were taken from the cornea by carefully scraping the ulcer with a Kimura platinum spatula (OPSM) or a sterile Bard-Parker scalpel blade. The first specimen was taken for culture and without local anaesthetic.

The patients with pneumococcal infection were treated with hourly irrigations of a penicillin solution, $200 \mathrm{units} / \mathrm{ml}$ in normal saline, administered with an intravenous giving set. Chloramphenicol ointment was used at night and penicillin 0.5 million units given subconjunctivally for four days as well as penicillin orally.

The Pseudomonas and other Gram-negative rod infections were treated with $1 \%$ gentamicin drops hourly, gentamicin $20 \mathrm{mg}$ subconjunctivally daily for four days, and polymyxin-oxytetracycline ointment at night.

The fungal ulcers were treated with $1 \%$ miconazole $(10 \mathrm{mg} / \mathrm{ml})$ drops second hourly, ${ }^{5}$ or in the case of Fusarium solani, which appears resistant to miconazole in vitro, pimafucin (Natamycin) drops $2.5 \%(25 \mathrm{mg} / \mathrm{ml})$ were given second hourly. Miconazole was also given as subconjunctival injections of $10 \mathrm{mg}$ daily for four days, with additional subconjunctival injections later in some cases.

\section{EPIDEMIOLOGY}

Sixteen rice grains from a batch of stored rice were cultured, eight on Sabouraud's agar and eight on blood agar, in an attempt to detect potential patho- 
gens. Seven samples of pond water and three samples of canal water from the Chittagong area were cultured on blood and Sabouraud's agar before and after 10 -fold concentration by centrifugation and colonies were enumerated approximately.

Conjunctival swabs were taken from healthy untreated eyes of four nursing staff and six cataract patients and cultured on blood and chocolate agar. Nasal swabs were taken from four doctors, four nursing staff, and eight patients and cultures examined for staphylococcal colonies. These were tested by slide coagulase to identify Staph. aureus.

\section{Results*}

The lightweight incubator functioned well with steady uniform temperature maintenance at $35^{\circ} \mathrm{C}$. The media made with tap water grew fastidious pathogens such as Str. pneumoniae and Haemophilus influenzae, including strains isolated directly from patients' eyes. The availability of small quantities of distilled water was necessary for preparing stains, but untreated tap water proved satisfactory for media and for washing slides.

The Gram stain proved adequate to detect fungi in those cases from which they were isolated, the main factor affecting detection being the adequacy of the scraping taken with the platinum spatula. It was noticed particularly with bacterial ulcers that organisms were best detected from the scrapings of

\footnotetext{
${ }^{*}$ During the period of study four additional patients were noted attending the hospital with recurrent herpes simplex keratitis with typical dendritic ulcers revealed by fluorescein staining in two cases and a geographic ulcer in a third case previously treated with steroidcontaining ointment, which is freely obtainable in the smallest of villages throughout Bangladesh. These patients were treated with vidarabine or idoxuridine ointment. Another inpatient, a young woman, had bilateral corneal scars with a history of recurrent ulceration self treated with steroids which was related to a history of cold sores on the mouth.
}

Table 1 Organisms detected

\begin{tabular}{ll}
\hline Organism & Number of Patients \\
\hline Bacteria & 5 \\
Streptococcus pneumoniae & 3 \\
Pseudomonas aeruginosa & 3 \\
Staphylococcus epidermidis & 1 \\
Enterobacter & 1 \\
Providencia & 3 \\
Unidentificd Gram-ncgative rods & 2 \\
Corynebacterium (normal flora) & \\
Fungi & 1 \\
Aspergillus fumigatus (Fig. 2) & 1 \\
Aspergillus ochraceus & 1 \\
Fusarium solani (Fig. 3) & 4 \\
Unidentificd filamentous fungi & \\
\hline
\end{tabular}

the cornea itself, and were frequently absent from the loose exudate overlying ulcers.

Contamination of agar plates with airborne bacteria or fungi could be distinguished from growth from the patient's specimen by the relationship of the colonies to the inoculating streak. That is, growth along the streaks was related to the patient, while growth away from the streaks was due to contamination. Airborne bacteria and fungi were relatively more common in Chittagong than in Sydney.

A history of initiating trauma was obtained in 14 of the 29 patients with suppurative keratitis. The commonest trauma was due to the husk of the rice grain flying into the eye during the process of beating the rice to remove the husks - that is, 'paddy injury'which was reported in seven cases. Other causes of trauma included sand (two boys), a date palm, a wooden stick, a bamboo splinter, and a needle. In the case of the pseudomonas keratitis, the cause was thought to be almost certainly due to contaminated water with which the injured eye was initially washed.

Scrapings taken from corneal ulcers of the 29 patients with suppurative keratitis revealed the following organisms in Gram stains and cultures (Table 1).

Gram stains and cultures did not reveal any significant micro-organisms in the other patients, though two had been treated in hospital for two and four weeks and others had purchased ointments, which probably included antibiotics in many cases, before coming to the BNSB Eye Infirmary.

Interestingly in three cases Gram stains revealed Gram-intermediate (i.e., pinky-blue) curved rods in corneal ulcer scrapings. They were found in cases of pneumococcal ulcer and in one case where only symbiotic Corynebacterium xerosis was isolated in culture. They were also seen in a smear from another

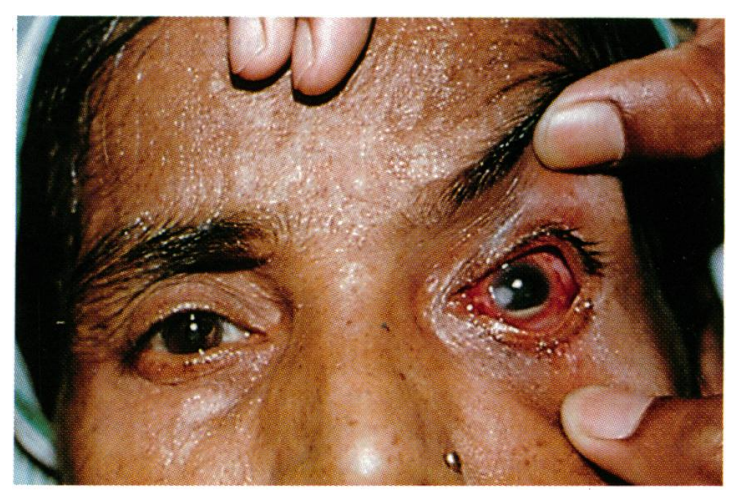

Fig. 2 A typical presentation of suppurative keratitis. $A$ fungal cause was not suspected until identified on Gram stain (characterised as Aspergillus fumigatus on culture). 


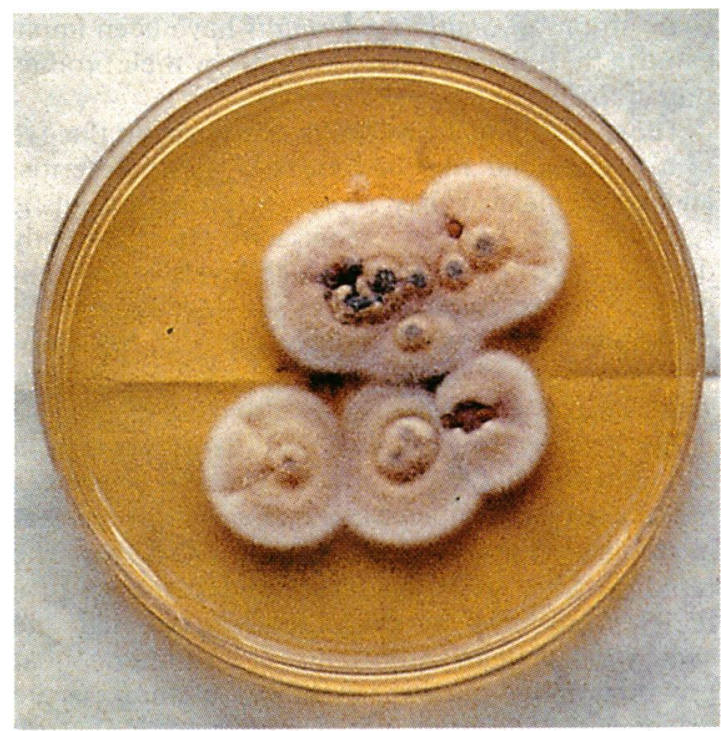

Fig. 3 Culture on Sabaraud's agar of colonies of Fusarium solani.

patient who had been in hospital for six weeks from whom a fungus was isolated which could not subsequently be identified owing to overgrowth by a contaminating fungus. No such bacteria could be grown in culture, though anaerobic cultures were not done and the organisms were reminiscent of the appearance of Mobiluncus, which is a Gram-variable anaerobic curved rod implicated as a cause of vaginosis. ${ }^{6}$

Also seen in Gram stains of scrapings from three patients were yeast-like organisms with capsules staining Gram-negative which did not grow in culture and were presumed to be Pityrosporum orbiculare. This is a yeast-type fungus associated particularly with seborrhoeic dermatitis and probably coming from the eyelashes as a harmless saphrophyte.' The patients mostly presented late with advanced corneal ulcers, and the appearance of the ulcer was not helpful in the differential diagnosis of bacterial and fungal infection. The commonest initiating trauma for corneal ulcers was due to husking rice (paddy

Table 2 Comparison of fungal and bacterial infections

\begin{tabular}{ll}
\hline Fungi & Bacterial \\
\hline Total 7 & 14 \\
Injury 1 (paddy grain) & $7(4$ paddy grain) \\
Male/female (4/3) & $12 / 2$ \\
Average duration* $2 \cdot 3$ weeks before presentation & 11 days \\
Average age 50 years & 30 years \\
\hline
\end{tabular}

*The duration before presentation was estimated from the time of injury if the patient could recall it, or was based on the duration of patients symptoms. The onset of symptoms in the bacterial cases tended to be more severe and resulted in early presentation. injury). Among the patients in whom fungi were found, only one had a history of paddy injury compared with four of 14 with bacterial infection. However, paddy grain injury was not associated with pneumococcal infection. Hence there was little in the history of patient characteristics to point to fungal or bacterial infections, except a longer history in some of the cases of fungal ulcers. Even the duration of ulceration prior to presentation was often similar in the fungal and bacterial cases, though four of the patients with fungal ulcers spent an average of 3.5 weeks in hospital receiving antibacterial therapy before this project started (Table 2).

The ulcers showed steady improvement with the treatment regimens described despite their advanced pathology at presentation, and all patients but one, who had the affected eye enucleated, were discharged with the disease apparently arrested.

\section{EPIDEMIOLOGY RESULTS}

All eight stored rice grains cultured on Sabouraud's agar and blood agar grew Bacillus species and six of the eight on Sabouraud's agar grew fungi of the family Mucorales. Five of the eight also grew a yellow Aspergillus species similar to that isolated from one patient. Two other unidentified fungi were also seen in the cultures.

Samples of pond and canal water, which are often used to wash injured eyes, were cultured. An average of $10^{3}$ to $10^{5}$ bacteria per $\mathrm{ml}$ were found, including Gram-negative rods in eight of 10 samples, yeast in two, and Bacillus species in two.

Conjunctival swabs from four healthy staff and six uninfected patients revealed occasional Staph. epidermidis in six and Corynebacterium species in two cases and, interestingly, Haemophilus influenzae in one outpatient presenting for refraction. A survey for Staph. aureus carriers revealed positive nose swabs from two of four doctors, one of four nursing staff or paramedics, and two of eight patients. All five isolates of Staph. aureus were resistant to penicillin.

\section{Discussion}

Our experience confirms that fungi are an important cause of suppurative keratitis in Bangladesh, accounting for some $30 \%$ of the cases of suppurative keratitis in this study. Furthermore we have found that there is a variety of fungi involved reminiscent of the diversity of genera found in (sub) tropical areas in the USA and elsewhere. ${ }^{x}$ In Florida the commonest cause of fungal ulceration is Fusarium solani, which has not previously been well recognised as a cause in Bangladesh. Also Aspergillus fumigatus and Aspergillus flavus and many other fungi have been reported in other countries. We found Aspergillus 
fumigatus as previously reported in Bangladesh ${ }^{3}$ and a sulphur yellow species that was probably Aspergillus ochraceus. This yellow species was also isolated from rice grains and may be common in the environment.

The pathogenesis of fungal keratitis is unclear. Trauma is assumed to be an initiating factor, though usually, as in our cases, there is no clear incident of injury. ${ }^{8}$ Bacterial and fungal organisms growing in the thatch of houses have been implicated as causing allergic pneumonia to people living in warm climates. ${ }^{9}$ Environmental cultures from dwellings of patients with fungal ulcers might be of interest, though two small random samples of thatch did not reveal fungi in this study.

The significance of the finding of fungi as a cause of suppurative keratitis is important when it is appreciated that antifungal therapy for suppurative keratitis is not freely available in Bangladesh. Furthermore, the problem of suppurative keratitis due to fungi is aggravated by the fact that corticosteroid ointments are freely available in the villages.

We found the Gram stain alone was of value in making a diagnosis of bacterial or fungal keratitis. In an extended study to look at this question we found that in a series of 66 cultures 58 were culture-positive for bacterial or fungal organisms, and, of these, 47 were anticipated on Gram stain examination alone.

Establishing the pattern of infective organisms causing suppurative keratitis was fundamental to developing appropriate treatment protocols. These protocols are referred to above in clinical methods. The results of these therapeutic regimens was one of resolution and healing in those cases with proved microbiological diagnosis, except for one case of pseudomonas infection which was too advanced at presentation and resulted in perforation of the cornea soon after treatment began. The fungal infections were treated with topical and subconjunctival miconazole, and this therapy was effective except in one case due to fusarium, which is known to be resistant to miconazole.

The frequency of Str. pneumoniae as a cause of corneal ulcers is of interest. In contrast to some reports ${ }^{10}$ a history of trauma was not obtained from many of our patients with pneumococcal ulcers. It is likely that many patients have pneumococcal colonisation of the throat and/or conjunctiva and that only occasionally the more virulent capsular serotypes of pneumococci, such as type 4 , cause keratitis.

The role of the coagulase-negative staphylococci grouped here as Staph. epidermidis in the corneal ulcers from which they were isolated is uncertain. These organisms are now separated into several species of varying degrees of parasitism. "However, some strains of Staph. epidermidis have been implicated in the pathogenesis of keratitis by their production of toxins. ${ }^{12}$

The isolation of Providencia species from the eye of a young child who had measles, gastroenteritis, and keratomalacia may represent an opportunistic relatively less virulent colonisation of an already damaged eye. The relatively high levels of environmental bacteria in canal and pond water suggest the likelihood of such water, which is needed for washing, as a source of colonisation of the injured cornea. Traditional eye treatments may also contribute. ${ }^{13}$

The finding of Pseudomonas aeruginosa in a patient with rapidly progressive keratitis reinforces the necessity for vigilance in the case of solutions that might be used to wash foreign bodies from eyes. The rapid progression of this infection depends on proteinases produced by the organisms,${ }^{14}$ though the exotoxin A appears important. ${ }^{15}$ It is of interest that pseudomonas elastase is a metallo-proteinase, as are collagenases, including that of neutrophils ${ }^{16}{ }^{17}$ that are inhibited by tetracycline, which binds the metallic radical in the enzyme. Thus the combination of polymyxin with oxytetracycline (Terramycin), which is the routine ointment for eye infections at BNSB hospitals, may be appropriate for pseudomonas keratitis, though the elastase enzyme appears to be less important than the alkaline protease. ${ }^{15}$ However, tetracycline is synergistic with amphotericin B against Aspergillus species. ${ }^{18}$ Thus the addition of pimaricin (which is related to amphotericin) to treatment with polymyxin-oxytetracycline may provide good antifungal prophylactic therapy.

Treatment regimens effective in this pilot study, including antifungal therapy with subconjunctival miconazole, yielded results that in our opinion are deserving of further study.

In conclusion we believe this work confirms the feasibility of setting up similar relatively cheap microbiology laboratory facilities to develop strategies for the treatment of suppurative keratitis, and to help reduce avoidable blindness in developing countries. The total cost of equipment, setting up the laboratory, and training of the microbiologist was approximately 10000 Australian dollars. Short of this, merely the facilities for doing Gram stain identification will allow appropriate treatment in the majority of cases, which will subsequently be shown to be positive to culture. The addition of this skill to the base hospitals in rural areas of Bangladesh is proposed with the Chittagong Eye Hospital and Training Complex remaining as the reference centre for problem cases.

A further study of treatment regimens will be the subject of another report when larger numbers of cases are available. ${ }^{*}$ 
${ }^{*}$ Following the setting up of this laboratory in 1983-4 Dr A Richards and Dr E D Wright brought protocols to Chittagong which modified the treatment regimens outlined. These protocols are being filled in by the staff of the laboratory, and this material will be the subject of a future ioint publication.

Foresight Australia funded the microbiology laboratory in Chittagong, and the training of A Howlader in Sydney. Thai International Airlines transported laboratory equipment from Sydney to Bangladesh without charge. Professor Y E Cossart and Mr L Taylor, of the Department of Infectious Diseases, University of Sydney, provided considerable assistance and advice. Medications were donated by Roussel Pharmaceuticals, Allergan, and Astra Pharmaceuticals and the Sydney Eye Hospital.

\section{References}

1 Wahed MA. Bacterial corneal ulcer. Trans Ophthalmol Soc Bangladesh 1981; 9: 9-11.

2 Rahman MD, Mustafizur. Management of fungal corneal ulcer. Trans Ophthalmol Soc Bangladesh 1981; 9: 12-9.

3 Jones DB, Liesegang TJ, Robinson NM. In: Washington JA, ed. Laboratory diagnosis of ocular infections. Cumitech 13. Washington, DC: American Society for Microbiology, 1981.

4 Bell SM. Antibiotic sensitivity testing by the CDS method. In: Hartwig N, ed. Clinical microbiology update programme, N 21 . Australian Society for Microbiology, 1984.

5 Foster CS. Miconazole therapy for keratomycosis. Am J Ophthalmol 1981; 91: 622-9.

6 Spiegel CA, Eschenbach DA, Amsel R, Holmes KK. Curved anaerobic bacteria in bacterial (nonspecific) vaginosis and their response to antimicrobial therapy. J Infect Dis 1983; 148: 817-22.

7 Fedukowicz HB. External infections of the eye. New York: Appleton Century Crofts, 1978.
8 Forster RK. Fungal diseases. In: Smolin G, ed. The cornea, basic science and clinical aspects. Boston: Little, Brown, 1983: 168-77.

9 Blackburn CRB, Green WF. Experimental extrinsic allergic alveolitis induced in the guinea-pig with Streptomyces olivaceus. Clin Allergy 1974; 4: 87-93.

10 Hyndiuk RA, Nassif KF, Burd EM. Bacterial diseases. In: Smolin, G, ed. The cornea, basic science and clinical aspects. Boston: Little, Brown, 1983: 147-68.

11 Klous WE, Schleifer KH. Simplified scheme for routine identification of human Staphylococcus species. J Clin Microbiol 1975; 1: 82-8.

12 Valenton MJ, Okumoto M. Toxin producing strains of Staphylococcus epidermidis. Arch Ophthalmol 1973; 89: 187.

13 Rahman MM, Razzaque MA. A microbiological approach to isolate micro-organisms from paddy grains and paddy leaves, snails, turmeric and rose water. Trans Ophthalmol Soc Bangladesh 1982; 10: 86-8.

14 Howe TR, Iglewski BH. Isolation and characterisation of alkaline protease-deficient mutants of Pseudomonas aeruginosa in vitro and in a mouse eye model. Infect Immun 1984; 43: 1058-63.

15 Ohman DE, Burns RP, Iglewski BH. Corneal infections in mice with toxin A and elastase mutants of Pseudomonas aeruginosa. $J$ Infect Dis 1980; 142: 547-55.

16 Brown SI, Hook CW, Tragakis MP. Presence, significance and inhibition of lysosomal proteoglycanase. Invest Ophthalmol Vis Sci 1972; 11: 149-52.

17 Hazlett LD, Berk RS. Effect of $\mathrm{C}_{3}$ depletion on experimental Pseudomonas aeruginosa ocular infection: Histopathological analysis. Infect Immun 1984; 43: 783-90.

18 Hughes CE, Harris C, Peterson LR, Gerding DN. Enhancement of the in vitro activity of amphotericin B against Aspergillus spp. by tetracycline analogs. Antimicrob Agents Chemother 1984; 26: 837-40.

Accepted for publication 30 May 1986. 